\title{
Exact NMR simulation of anti-cancer nano drug-DNA/ RNA complexes in gum cancer cells spin systems using tensor train formalism
}

\author{
Alireza Heidari ${ }^{1,2 *}$ and Ricardo Gobato ${ }^{3}$ \\ ${ }^{1}$ Faculty of Chemistry, California South University, 14731 Comet St. Irvine, CA 92604, USA \\ ${ }^{2}$ American International Standards Institute, Irvine, CA 3800, USA \\ ${ }^{3}$ Green Land Landscaping and Gardening, Seedling Growth Laboratory, 86130-000, Parana, Brazil
}

\begin{abstract}
In the current study, exact NMR simulation of anti-cancer Nano drug-DNA/RNA complexes in gum cancer cells spin systems using tensor train formalism are considered. Looking into the future, we would predict three promising directions. One is the rapid histology based on two-color exact NMR simulation of anti-cancer Nano drug-DNA/RNA complexes in gum cancer cells spin systems using tensor train formalism which can be used in operation room during gum cancer surgery. The second is in situ molecule-based diagnosis using handheld fast exact NMR simulation of anti-cancer Nano drug-DNA/RNA complexes in gum cancer cells spin systems using tensor train formalism, for example, handheld exact NMR simulation of anti-cancer Nano drug-DNA/RNA complexes in gum cancer cells spin systems using tensor train formalism or hyperspectral exact NMR simulation of anti-cancer Nano drug-DNA/RNA complexes in gum cancer cells spin systems using tensor train formalism. The third is multimodal imaging and spectroscopy system that integrate advantages of each modality and may offer better diagnosis for gum cancer.
\end{abstract}

\section{Introduction}

With the capability of label-free and highly sensitive analysis of biomolecules in situ, exact NMR simulation of anti-cancer Nano drugDNA/RNA complexes in gum cancer cells spin systems using tensor train formalism-based techniques offer robust tools for gum cancer diagnosis. Using fiber-optic-based light delivery and collection, exact NMR simulation of anti-cancer Nano drug-DNA/RNA complexes in gum cancer cells spin systems using tensor train formalism-based techniques are mostly performed on accessible tissue surfaces, for example, on the skin, in gastrointestinal tract, or intraoperatively. The strength of exact NMR simulation of anti-cancer Nano drug-DNA/ RNA complexes in gum cancer cells spin systems using tensor train formalism lies in the high sensitivity and specificity, which leads to fast and accurate differentiation between malignant or premalignant from normal tissues. The challenge of gum cancer diagnosis using exact NMR simulation of anti-cancer Nano drug-DNA/RNA complexes in gum cancer cells spin systems using tensor train formalism would still be how to find very specific molecular marker for different types of human gum cancers. Hyperspectral exact NMR simulation of anticancer Nano drug-DNA/RNA complexes in gum cancer cells spin systems using tensor train formalism, which can quantitatively map different species of molecules, is a good way to discover new molecular markers for gum cancer diagnosis [1-10].

\section{Results and discussion}

Exact NMR simulation of anti-cancer Nano drug-DNA/RNA complexes in gum cancer cells spin systems using tensor train formalism has long been used to analyze chemical compositions in biological systems. Owing to its high chemical specificity and noninvasive detection capability, exact NMR simulation of anti-cancer Nano drug-DNA/RNA complexes in gum cancer cells spin systems using tensor train formalism has been widely employed in gum cancer screening, diagnosis, and intraoperative surgical guidance in the past ten years. In order to overcome the weak signal of spontaneous exact NMR simulation of anti-cancer Nano drug-DNA/RNA complexes in gum cancer cells spin systems using tensor train formalism, coherent exact NMR simulation of anti-cancer Nano drug-DNA/ RNA complexes in gum cancer cells spin systems using tensor train formalism and exact NMR simulation of anti-cancer Nano drugDNA/RNA complexes in gum cancer cells spin systems using tensor train formalism have been developed and recently applied in the field of gum cancer research. This review focuses on innovative studies of the use of exact NMR simulation of anti-cancer Nano drug-DNA/ RNA complexes in gum cancer cells spin systems using tensor train formalism in gum cancer diagnosis and their potential to transition from bench to bedside (Figures 1 and 2).

*Correspondence to: Alireza Heidari, Faculty of Chemistry, California South University, 14731 Comet St. Irvine, CA 92604; American International Standards Institute, Irvine, CA 3800, USA, E-mail: Scholar.Researcher.Scientist@gmail. com; Alireza.Heidari@calsu.us; Central@aisi-usa.org

Key words: NMR, simulation, anti-cancer nano drug, DNA/RNA, complexes, gum cancer cells, spin, tensor, formalism

Received: August 22, 2020; Accepted: September 07, 2020, Published: September 11, 2020 


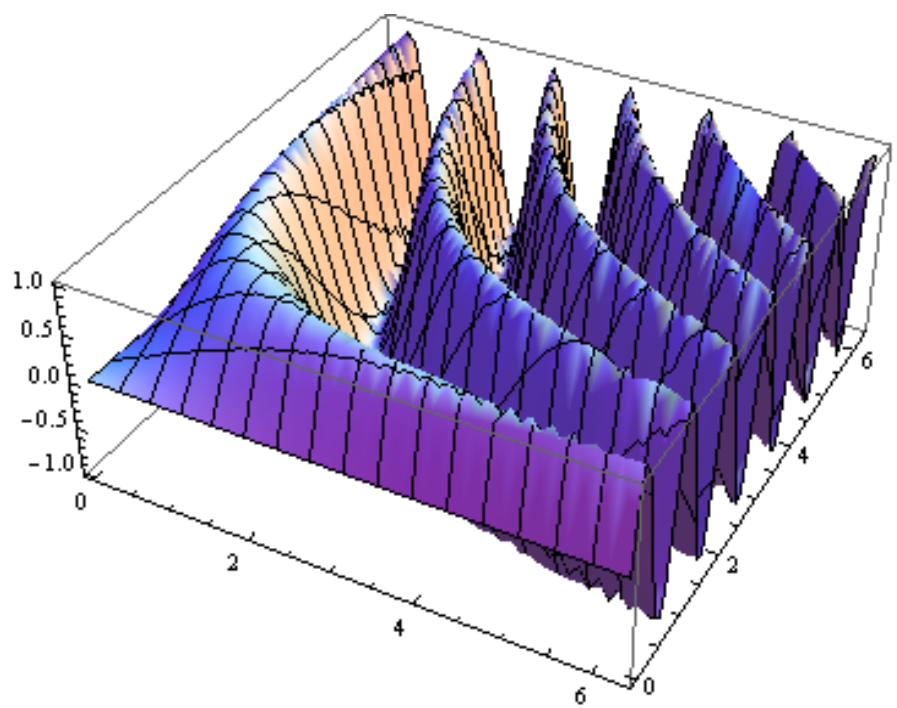

Figure 1. Exact NMR simulation of anti-cancer Nano drug-DNA/RNA complexes in gum cancer cells spin systems using tensor train formalism

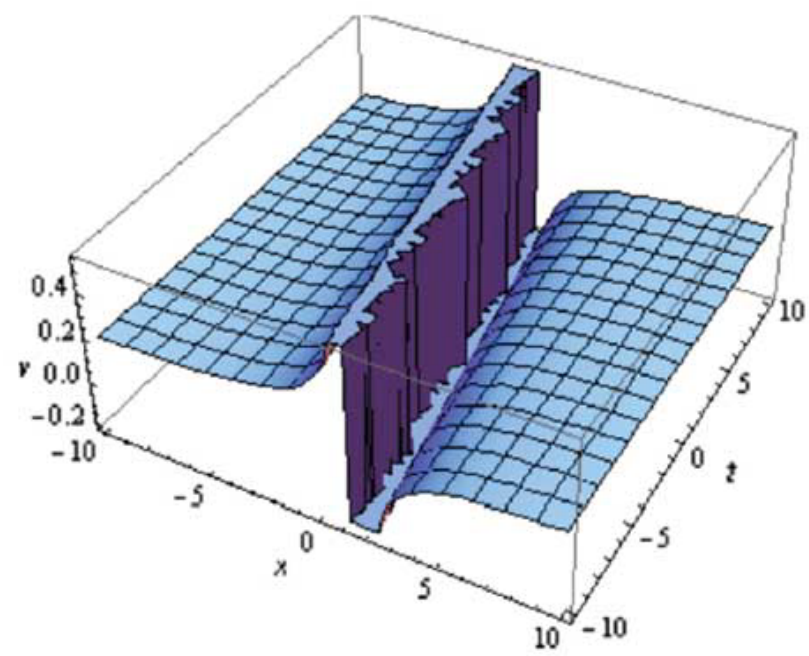

Figure 2. Exact NMR modelling of anti-cancer Nano drug-DNA/RNA complexes in gum cancer cells spin systems using tensor train formalism

\section{Conclusion}

Novel approaches toward understanding the evolution of disease can lead to the discovery of biomarkers that will enable better management of disease progression and improve prognostic evaluation. exact NMR simulation of anti-cancer Nano drug-DNA/RNA complexes in gum cancer cells spin systems using tensor train formalism is a promising investigative and diagnostic tool that can assist in uncovering the molecular basis of disease and provide objective, quantifiable molecular information for diagnosis and treatment evaluation. This technique probes molecular vibrations/rotations associated with chemical bonds in a sample to obtain information on molecular structure, composition, and intermolecular interactions. Exact NMR simulation of anti-cancer Nano drug-DNA/RNA complexes in gum cancer cells spin systems using tensor train formalism occurs when light interacts with a molecular vibration/rotation and a change in polarizability takes place during molecular motion. This results in light being scattered at an optical frequency shifted (up or down) from the incident light. By monitoring the intensity profile of the in elastically scattered light as a function of frequency, the unique spectroscopic fingerprint of a tissue sample is obtained. Since each sample has a unique composition, the spectroscopic profile arising from exact NMR simulation of anti-cancer Nano drug-DNA/RNA complexes in gum cancer cells spin systems using tensor train formalism-active functional groups of nucleic acids, proteins, lipids, and carbohydrates allows for the evaluation, characterization, and discrimination of tissue type. This review provides an overview of the theory of exact NMR simulation of anti-cancer Nano drug-DNA/RNA complexes in gum cancer cells spin systems using tensor train formalism, instrumentation used for measurement, and variation of exact NMR simulation of anti-cancer Nano drug-DNA/RNA complexes in gum cancer cells spin systems using tensor train formalism for clinical applications in gum cancer, including detection of brain, ovarian, breast, prostate, and pancreatic gum cancers and circulating tumor cells.

\section{Acknowledgments}

This study was supported by the Cancer Research Institute (CRI) Project of Scientific Instrument and Equipment Development, the National Natural Science Foundation of the United Sates, the International Joint BioSpectroscopy Core Research Laboratory Program supported by the California South University (CSU), and the Key project supported by the American International Standards Institute (AISI), Irvine, California, USA.

\section{References}

1. Heidari A, Brown C (2015) Study of composition and morphology of cadmium oxide (CdO) nanoparticles for eliminating cancer cells. J Nanomed Res 2(5): 20.

2. Heidari A, Brown C (2015) Study of surface morphological, phytochemical and structural characteristics of rhodium (III) oxide $\left(\mathrm{Rh}_{2} \mathrm{O}_{3}\right)$ nanoparticles. International Journal of Pharmacology, Phytochemistry and Ethnomedicine 1(1): 15-19.

3. Heidari A (2016) An experimental biospectroscopic study on seminal plasma in determination of semen quality for evaluation of male infertility. Int $J$ Adv Technol 7: e007.

4. Heidari A (2016) Extraction and preconcentration of N-Tolyl-Sulfonyl-PhosphoramidSaeure-Dichlorid as an anti-cancer drug from plants: a pharmacognosy study. $J$ Pharmacogn Nat Prod 2: e103.

5. Heidari A (2016) A thermodynamic study on hydration and dehydration of DNA and RNA-Amphiphile Complexes. J Bioeng Biomed Sci S: 006.

6. Heidari A (2016) Computational studies on molecular structures and carbonyl and ketene groups' effects of singlet and triplet energies of Azidoketene $\mathrm{O}=\mathrm{C}=\mathrm{CH}-\mathrm{NNN}$ and Isocyanatoketene $\mathrm{O}=\mathrm{C}=\mathrm{CH}-\mathrm{N}=\mathrm{C}=\mathrm{O} . J$ Appl Computat Math 5: e142.

7. Heidari A (2016) Study of irradiations to enhance the induces the dissociation of hydrogen bonds between peptide chains and transition from helix structure to random coil structure using ATR-FTIR, Raman and 'HNMR Spectroscopies. J Biomol Res Ther 5: e146.

8. Heidari A (2016) Future prospects of point fluorescence spectroscopy, fluorescence imaging and fluorescence endoscopy in photodynamic therapy (PDT) for cancer cells. J Bioanal Biomed 8: e135.

9. Heidari A (2016) A bio-spectroscopic study of DNA density and color role as determining factor for absorbed irradiation in cancer cells. Adv Cancer Prev 1: e102.

10. Heidari A (2016) Manufacturing process of solar cells using cadmium oxide (CdO) and rhodium (III) Oxide $\left(\mathrm{Rh}_{2} \mathrm{O}_{3}\right)$ nanoparticles. J Biotechnol Biomater 6: e125.

Copyright: (C2020 Heidari A. This is an open-access article distributed under the terms of the Creative Commons Attribution License, which permits unrestricted use, distribution, and reproduction in any medium, provided the original author and source are credited. 